\title{
Valvuloplastía Aórtica con balón como puente para reemplazo valvular aórtico percutáneo o quirúrgico en el manejo de la estenosis aórtica severa
}

\author{
Gabriel Maluenda, Itsik Ben-Dor, Israel Barbash, Dani Dvir, Petros Okubagzi, Rebecca Torguson, Rahewana Amare, Zhenyi Xue, \\ Joseph Lindsay, Lowell F. Satler, Ron Waksman, Augusto Pichard. \\ Division of Cardiology, Washington Hospital Center, Washington, DC
}

Introducción: La incorporación del reemplazo valvular aórtico percutáneo (RVAP) ha 'revivido' el uso de la valvuloplastía Aórtica con balón (VAB) para el tratamiento de la estenosis Aórtica (EA) severa sintomática.

Objetivos: Determinar la tasa de éxito y complicaciones después de VAB y los resultados clínicos como terapia única versus VAB como puente para RVA quirúrgico o percutáneo.

Métodos: La cohorte quedó compuesta por 472 pacientes que fueron sometidos a $538 \mathrm{VAB}$. En el grupo I, VAB se usó como terapia única, $\mathrm{n}=387(81.9 \%)$, y grupo II, VAB como puente, $\mathrm{n}=85(18.1 \%, \mathrm{n}=65$ a RVAP, $\mathrm{n}=20$ RVA quirúrgico). VAB exitosa fue definida como una reducción $>40 \%$ en gradiente aórtico medio y/o incremento en área valvular aórtica $>40 \%$.

Resultados: Los grupos I y grupo II fueron comparables en cuanto a edad $(81.7 \pm 8.3$ vs. $83.2 \pm 10.9$ años, $\mathrm{p}=0.18)$, o STS score $(13.1 \pm 6.2$ vs. $12.4 \pm 6.4, \mathrm{p}=0.4)$, o
Euroscore logístico ( $45.4 \pm 22.3$ vs. $46.9 \pm 21.8, \mathrm{p}=0.43$ ). El incremento medio en el área valvular Aórtica fue de $0.39 \pm 0.25 \mathrm{~cm} 2$ en el grupo I y $0.42 \pm 0.26 \mathrm{~cm} 2$ en el grupo II, $\mathrm{p}=0.33$. El descenso en la gradiente media fue de $24.1 \pm 13.1 \mathrm{mmHg}$ en el grupo I y de $27.1 \pm 13.8 \mathrm{mmHg}$ en el grupo II, $\mathrm{p}=0.06$. La VAB no fue exitosa en 81 casos (15\%). Por análisis multivariado se comprobó que la repetición de $\mathrm{VAB}$ era el correlato más potente para VAB no exitosa (HR 4.34[95\%, IC 2.2-8.3], $\mathrm{p}<0.001$ ). La mortalidad alejada fue de $55.2 . \%$ (214 pacientes) en el grupo I y de $22.3 \%(\mathrm{n}=19)$ en grupo II, $\mathrm{p}<0.001$. No hubo diferencias en muerte intra-procedimiento, $n=6$ (2\%) vs. $n=5(2 \%)$, o accidente cerebro vascular, $n=7$ $(2.3 \%)$ vs $n=4(1.6 \%)$.

Conclusión: En pacientes de alto riesgo con EA severa y contraindicación temporal para RVA quirúrgico/ percutáneo, la VAB puede ser usada como puente para una intervención definitiva con buen resultado a mediano plazo. 


\section{Percutaneous aortic balloon valvuloplasty as a bridge to aortic valve replacement in severe aortic stenosis}

Background: the recent introduction of percutaneous aortic valve replacement (PAVR) has become a stimulus to perform percutaneous aortic balloon valvuloplasty (PABV) in patients with severe symptomatic aortic stenosis (AS) as a bridge to valve replacement (AVR)

Aim: to determine success rates and clinical results of PABV alone vs those obtained with PABV followed by either surgical or percutaneous AVR.

Method: 472 patients with severe AS underwent a total of $538 \mathrm{PABV}$ procedures. 378 (82\%) were treated with PABV alone (Group I). In Group II, 85 patients $(18 \%)$ had PABV followed by either PAVR $(\mathrm{n}=65)$ or surgical AVR ( $\mathrm{n}=20)$. A successful PABV was defined as $>40 \%$ reduction in mean aortic valve pressure gradient or $>40 \%$ increase in aortic valve area

Results: Groups I and II were comparable regarding age $(81.7 \pm 8.3$ vs. $83.2 \pm 10.9$ years, $\mathrm{p}=0.18)$,

\section{Introducción:}

El reemplazo valvular aórtico (RVA) quirúrgico ha sido establecido como un tratamiento efectivo para la estenosis Aórtica (EA) severa sintomática. ${ }^{1}$ El RVA percutáneo transcateter ha emergido como una nueva alternativa para el tratamiento de la EA severa en pacientes de riesgo quirúrgico muy alto $^{2}$ y para pacientes considerados inoperables ${ }^{3}$. Sin embargo, como previamente reportado ${ }^{4,5}$ hasta un $75 \%$ de los pacientes que son evaluados como potenciales candidatos a RVA percutáneos no son elegibles para esta terapia y muchos de ellos son referidos para valvuloplastía Aórtica con balón (VAB).

El entusiasmo inicial de la VAB, inicialmente introducida por Cribier et al. en $1986{ }^{6}$ posteriormente disminuyó por estudios que demostraron que si bien proveía de un beneficio sintomático inicial, ${ }^{7}$ estaba con frecuencia asociado a altas tasas de complicaciones y de recurrencia, ${ }^{8}$ con poco impacto en la sobrevida a largo plazo, y por tanto su empleo disminuyó considerablemente ${ }^{9}$ Después de la introducción de RVA percutáneo trans-catéter se ha observado un importante resurgimiento del procedimiento de VAB. En nuestra institución, el número de VAB realizadas se ha incrementado de un 4.4 casos/año a 88.6 casos/año después de la introducción de RVA percutáneo..$^{10} \mathrm{La}$ VAB puede ser empleada como una terapia única para el alivio
STS score (13.1 \pm 6.2 vs. $12.4 \pm 6.4, \mathrm{p}=0.4)$ and Logistic Euroscore $(45.4 \pm 22.3$ vs. $46.9 \pm 21.8, \mathrm{p}=0.43)$. Mean increase in AVA was $0.39 \pm 0.25 \mathrm{~cm} 2$ in Group I and $0.42 \pm 0.26 \mathrm{~cm} 2$ in Group II ( $\mathrm{p}=0.33$ ). Mean aortic valve pressure gradient decreased $24.1 \pm 13.1 \mathrm{mmHg}$ in Group I and $27 \pm 1.8 \mathrm{mmHg}$ in Group II ( $\mathrm{p}=0.06)$. PABV was not successful in 81 patients $(15 \%)$. As expected, repeated PABV was the main predictor for failure (HR 4.34[95\%, CI 2.2-8.3], p<0.001). Midterm mortality rate was $55.2 \%$ (214 patients) in Group I and $22.3 \%$ (19 patients) in Group II ( $\mathrm{p}<0.001)$. Intra-procedure mortality was identical in both Groups (2\%). Stroke occurred in $2.3 \%$ of patients in Group I vs $1.6 \%$ in Group II.

Conclusion: In high risk patients with severe AS and temporary contraindication for percutaneous or surgical AVR, PABV may be used as a bridge for intervention with good midterm results.

sintomático o bien como medida temporal en pacientes que esperan un implante valvular definitivo. La VAB es empleada como 'puente' a RVA quirúrgico/percutáneo en paciente hemodinámicamente inestables, o en pacientes con contraindicación temporal para RVA debido a disfunción sistólica, hipertensión pulmonar, insuficiencia mitral, enfermedad pulmonar o falla renal muy severa $y / o$ fragilidad extrema. Este estudio tiene por objetivo determinar la tasa de éxito y de complicaciones de la VAB y los resultados alejados de la VAB como terapia única o como puente a un RVA quirúrgico/percutáneo.

\section{Métodos:}

Población de estudio. Desde Enero 2006 a Mayo 2011, los datos de 538 procedimientos consecutivos de VAB correspondientes a 472 pacientes con EA severa sintomática fueron entrados a una base de datos dedicada. Todos los pacientes tenían EA severa sintomática confirmada por ecocardiograma trans-torácico y evaluación hemodinámica. Los pacientes fueron referidos para paliación de síntomas de falla cardíaca, tratamiento de shock cardiogénico o como puente a RVA quirúrgico/percutáneo. Esta cohorte se dividió en 2 grupos: grupo 1, pacientes sometidos a VAB como terapia única, $\mathrm{n}=387(81.9 \%)$; y grupo 2 , pacientes sometidos a VAB como puente, $\mathrm{n}=85(18.1 \%$, 
n=65 -RVA percutáneo, n=20 -RVA quirúrgico).

Los índices de riesgo STS (Society of Thoracic Surgeons) y EuroSCORE logístico fueron calculados en todos los pacientes. Los eventos intra-hospitalarios fueron determinados por revisión de registros médicos. El seguimiento clínico fue realizado por personal con entrenamiento médico vía entrevista telefónica o en visitas médicas ambulatorias. Las fuentes primarias de documentación de cada evento clínico fueron revisadas por personal médico independiente, quienes determinaron la naturaleza de los eventos.

Datos Ecocardiográficos: Todos los pacientes fueron evaluados por ecocardiografistas expertos utilizando sistemas de ultrasonido comercialmente disponibles. Ecocardiografía convencional en modo $\mathrm{M}$, bidimensional y con Doppler-color fueron registrados antes y después de la VAB. Se emplearon múltiples posiciones del transductor a fin de asegurar medición precisa según jet de velocidad aórtica máxima. El área valvular Aórtica (AVA) fue calculada usando la ecuación de continuidad.

Procedimiento: El procedimiento de VAB fue realizado usando a técnica vía retrograda por acceso femoral. Se usó heparina no fraccionada en todos los pacientes en dosis de 10-70 U/Kg después de la inserción de introductor arterial de 8-13F. Las presiones medidas en la raíz Aórtica y brazo lateral del introductor femoral fueron igualadas antes de intentar cruce a ventrículo izquierdo. Las presiones fueron registradas durante retiro a través de la válvula aórtica. Se obtuvieron los gradientes transvalvulares máximos y medios junto a gasto cardíaco por termo-dilución antes y después de la VAB. El AVA fue calculada usando gradientes medios según método de Gorlin.

A fin de estabilizar la posición del balón a través de la válvula aórtica, se realizó pacing ventricular derecho a alta frecuencia (180-200 lpm) antes de insuflación hasta lograr una caída en la presión sistémica $<50 \mathrm{~mm} \mathrm{Hg}$. La VAB fue considerada adecuada cuando la gradiente media disminuyó $>30-40 \%$ del valor basal. Cuando esto no fue logrado tras primer intento, se realizaron insuflaciones adicionales, y se usaron balones de mayor tamaño en casos seleccionados. El acceso arterial fue cerrado usando sistemas de cierre percutáneo: Perclose 6F (Abbott Vascular, Abbott Park, IL), Prostar 10F (Abbott Vascular), o Angio Seal 8F (St Jude Medical, St. Paul, MN), y compresión manual en caso de fallo de cierre.

Definiciones: VAB exitosa fue definida como una reducción en la gradiente media y/o incremento en el área valvular Aórtica en más de $40 \%$. Evento adverso serio fue definido como muerte intra-procedimiento y/o accidente vascular cerebral (AVC) después de VAB. Otros eventos registrados incluyeron insuficiencia Aórtica moderada a severa, hipotensión profunda que requirió resucitación o intubación o cardioversión, taponamiento cardíaco, implante de marcapaso permanente y complicación vascular del acceso que requirió intervención. Los eventos intrahospitalarios fueron determinados por revisión de registros médicos. Todos los pacientes fueron seguidos en forma telefónica o en visita ambulatoria.

Análisis estadístico: Las variables continuas son presentadas como media \pm desviación estándar (DE) y las variables categóricas como valor absoluto y porcentajes. Los tiempos de seguimiento son presentados como mediana $\mathrm{e}$ intervalo intercuartil (percentil 25-75\%). Las diferencias entre variables continuas fueron evaluadas por test $\mathrm{T}$ de Student. Las variables categóricas fueron comparadas usando test de Chi-cuadrado o test exacto de Fisher según corresponda. Se realizaron curvas de sobrevida acumulada usando método de Kaplan-Meier y comparados por test de log-rank. Para identificar las variables correlacionadas con mortalidad se realizó un análisis univariado seguido de un análisis multivariado de Cox con metodología de 'stepwise' para aquellas variables con valor $\mathrm{p}<0.05$. Se realizó un análisis univariado seguido por análisis de regresión logística para identificar las variables correlacionadas con éxito de VAB. Todas las variables clínicas, ecocardiográficas y hemodinámicas asociadas a éxito de VAB con valor $\mathrm{p} \leq 0.2$ en análisis univariado fueron entradas en modelo de regresión logístico. Valor de significancia p fue establecido como $<0.05$.

\section{Resultados:}

Procedimientos: La cohorte quedó compuesta por 472 individuos consecutivos que fueron sometidos a 538 procedimientos de VAB. De estos pacientes, 51 recibieron 2 procedimientos de $\mathrm{BAV}, 12$ recibieron $3 \mathrm{VAB}, 2$ recibieron $4 \mathrm{VAB}$ y un paciente recibió $5 \mathrm{VAB}$.

Características basales: No existieron diferencias significativas en términos de las características demográficas o comorbilidades entre pacientes tratados con VAB como terapia única o como puente para RVA, como se aprecia en la Tabla 1. Específicamente, no hubo diferencias entre grupos 1 vs. 2 en términos de edad media, score STS, Euroscore logístico, u otras comorbilidades. En términos de las variables ecocardiográficas y hemodinámicas, los pacientes sometidos a VAB como puente tuvieron gradientes medios transvalulares más elevados así como un área valvular de menor tamaño comparados contra aquellos sometidos a VAB como terapia única (Tabla 2). En la misma tabla se observa que no hubo diferencias significativas entre ambos grupos en términos de la función ventricular 
izquierda así como las presiones cardíacas derechas.

Datos de procedimiento: Los detalles del procedimiento son presentados en la Tabla 3. Ambos grupos tuvieron similares grados de reducción del gradiente aórtico medio (23.1 $\pm 13.5 \mathrm{~mm}$ de $\mathrm{Hg}$ en grupo 1vs. $25.4 \pm 13.7 \mathrm{~mm} \mathrm{de} \mathrm{Hg}$ en grupo $2, \mathrm{P}=0.13$ ) e incremento del área valvular aórtica $(0.38 \pm 0.25 \mathrm{~cm} 2$ en grupo 1 y $0.40 \pm 0.27 \mathrm{~cm} 2$ en grupo $2, \mathrm{p}=0.53)$. Sin embargo los pacientes sometidos a VAB como 'puente' recibieron con mayor frecuencia angioplastía coronaria concomitante comparado contra aquellos tratados con BAV como intervención única $(16,3 \%$ vs. $26.2 \%, p=0.01)$, aunque con similares cantidades de contraste.

\begin{tabular}{|c|c|c|c|}
\hline \multicolumn{4}{|c|}{ Tabla I: Características basales } \\
\hline Variable & $\begin{array}{c}\text { VAB } \\
(n=387)\end{array}$ & $\begin{array}{l}\text { VAB 'puente' } \\
\quad(n=85)\end{array}$ & $\mathbf{P}$ \\
\hline Edad (años) & $81.7 \pm 8.3$ & $83.2 \pm 10.9$ & 0.18 \\
\hline Sexo masculino (\%) & $172(44.4)$ & $38(44.7)$ & 0.96 \\
\hline Score de Society of & $13.1 \pm 6.2$ & $12.4 \pm 6.4$ & 0.41 \\
\hline Thoracic Surgeons (\%) & & & \\
\hline EuroSCORE Logístico & $45.4 \pm 22.3$ & $46.9 \pm 21.8$ & 0.43 \\
\hline Shock cardiogénico & $29(7.5)$ & $6(7.05)$ & 0.92 \\
\hline Clase funcional IV & $268(69.2)$ & $67(78.8)$ & 0.07 \\
\hline Diabetes Mellitus & $129(33.3)$ & $33(38.8)$ & 0.33 \\
\hline Hipertensión Arterial & $324(83.7)$ & 78(91.7) & 0.06 \\
\hline Enfermedad coronaria (\%) & $238(61.4)$ & $52(61.7)$ & 0.95 \\
\hline CRM previa (\%) & $107(27.4)$ & $23(27.0)$ & 0.93 \\
\hline Enf. Pulmonar obstructiva crónica & 96(24.8) & $14(16.4)$ & 0.09 \\
\hline Falla renal crónica (TFG $<60 \mathrm{~m} / / \mathrm{min}$ ) & $171(44.1)$ & $35(41.1)$ & 0.61 \\
\hline AVC/TIA previo & $79(20.4)$ & 18(21.1) & 0.87 \\
\hline Fibrilación auricular & $137(35.4)$ & 21(24.7) & 0.06 \\
\hline Enf. Vascular periférica & $129(33.3)$ & $23(27.0)$ & 0.26 \\
\hline
\end{tabular}

VAB, valvuloplastía Aórtica con balón; CRM: cirugía de revascularización coronaria; $T F G$, tasa de filtración glomerular

\begin{tabular}{|c|c|c|c|}
\hline Variable & $\begin{array}{c}\text { VAB } \\
(n=387)\end{array}$ & $\begin{array}{l}\text { VAB 'puente' } \\
\qquad(\mathrm{n}=85)\end{array}$ & $\mathbf{P}$ \\
\hline \multicolumn{4}{|l|}{ Parámetros ecocardiográficos } \\
\hline Fracción de eyección (\%) & $45.1 \pm 17.8$ & $45.8 \pm 17.4$ & 0.83 \\
\hline Área valvular Aórtica (cm2) & $0.69 \pm 0.15$ & $0.65 \pm 0.17$ & 0.02 \\
\hline Velocidad máxima de jet (m/seg) & $3.9 \pm 0.68$ & $4.3 \pm 0.70$ & $<0.001$ \\
\hline Gradiente valvular aórtico medio (mm $\mathrm{Hg})$ & $42.1 \pm 28.5$ & $49.0 \pm 15.3$ & $<0.001$ \\
\hline Presión sistólica de arteria pulmonar ( $(\mathrm{mm} \mathrm{Hg}$ ) & $53.2 \pm 16.8$ & $50.1 \pm 16.2$ & 0.19 \\
\hline \multicolumn{4}{|l|}{ Parámetros hemodinámicos } \\
\hline Área valvular Aórtica (cm2) & $0.61 \pm 0.21$ & $0.57 \pm 0.18$ & 0.11 \\
\hline Gradiente valvular aórtico medio $(\mathrm{mm} \mathrm{Hg}$ ) & $44.4 \pm 19.7$ & $52.7 \pm 18.5$ & $<0.001$ \\
\hline Presión de auricula derecha $(\mathrm{mm} \mathrm{Hg})$ & $10.0 \pm 5.2$ & $10.1 \pm 4.8$ & 0.92 \\
\hline Presión sistólica de arteria pulmonar $(\mathrm{mm} \mathrm{Hg})$ & $54.8 \pm 16.4$ & $53.6 \pm 15.3$ & 0.57 \\
\hline Presión de capilar pulmonar (mm Hg) & $21.3 \pm 8.6$ & $23.1 \pm 6.4$ & 0.20 \\
\hline Gasto cardiaco (I/min) & $4.0 \pm 1.3$ & $4.1 \pm 1.2$ & 0.39 \\
\hline
\end{tabular}

$V A B$, valvuloplastía aórtica con balón

\begin{tabular}{|c|c|c|c|}
\hline Variable & $\begin{array}{c}\text { VAB } \\
(n=434)\end{array}$ & $\begin{array}{l}\text { VAB 'puente' } \\
(n=103)\end{array}$ & $\mathbf{P}$ \\
\hline \multicolumn{4}{|l|}{ Procedimiento } \\
\hline Tiempo de radioscopia (min) & $14.5 \pm 8.3$ & $13.3 \pm 6.8$ & 0.16 \\
\hline Angioplastia concomitante & $71(16.3 \%)$ & $27(26.2 \%)$ & 0.01 \\
\hline Cantidad de contraste (cc) & $53.1 \pm 48$ & $43.1 \pm 49$ & 0.23 \\
\hline Dosis de heparina (Unidades) & $2543 \pm 1521$ & $2669 \pm 1587$ & 0.56 \\
\hline \multicolumn{4}{|l|}{ Tamaño del introductor } \\
\hline $8 \mathrm{~F}$ & $17(3.9 \%)$ & 0 & \\
\hline $9 \mathrm{~F}$ & $36(8.3 \%)$ & $13(12.6 \%)$ & \\
\hline $10 \mathrm{~F}$ & $190(43.7 \%)$ & $56(54.3 \%)$ & NS \\
\hline $12 \mathrm{~F}$ & $115(26.4 \%)$ & $25(24.3 \%)$ & \\
\hline $13 \mathrm{~F}$ & $75(17.2 \%)$ & $9(8.7 \%)$ & \\
\hline \multicolumn{4}{|l|}{ Tamaño máximo de balón } \\
\hline $18 \mathrm{~mm}$ & $3(0.7 \%)$ & 0 & \\
\hline $20 \mathrm{~mm}$ & $73(16.8 \%)$ & $12(11.6 \%)$ & \\
\hline $22 \mathrm{~mm}$ & $127(29.2 \%)$ & $37(21.3 \%)$ & NS \\
\hline $23 \mathrm{~mm}$ & $66(15.3 \%)$ & $14(13.5 \%)$ & \\
\hline $25 \mathrm{~mm}$ & $171(39.4 \%)$ & $40(24.2 \%)$ & \\
\hline Relación tamaño anillo/balón & $1.15 \pm 0.13$ & $1.14 \pm 0.09$ & 0.59 \\
\hline Más de una insuflación & $222(51.1)$ & $44(42.7 \%)$ & 0.12 \\
\hline Más de 2 insuflaciones & $55(12.6)$ & $14(13.6 \%)$ & 0.78 \\
\hline Disminución gradiente medio (mm Hg) & $23.1 \pm 13.5$ & $25.4 \pm 13.7$ & 0.13 \\
\hline Incremento medio en área valvular (cm2) & $0.38 \pm 0.25$ & $0.40 \pm 0.27$ & 0.53 \\
\hline Éxito de VAB & $361(85.3 \%)$ & $82(80.4 \%)$ & 0.47 \\
\hline \multicolumn{4}{|l|}{ Curso intra-hospitalario } \\
\hline Disminución de hemoglobina (mg \%) & $1.6 \pm 1.3$ & $1.4 \pm 1.1$ & 0.38 \\
\hline Disminución de hematocrito (\%) & $5.0 \pm 4.2$ & $4.8 \pm 3.2$ & 0.53 \\
\hline Incremento en creatinina (mg \%) & $0.39 \pm 1.0$ & $0.16 \pm 0.37$ & $<0.001$ \\
\hline Aumento de creatinina $>\mathbf{5 0} \%$ & $42(9.6 \%)$ & $7(6.7 \%)$ & 0.36 \\
\hline Incremento en niveles de troponinas ( $\mathrm{ng} / \mathrm{ml}$ ) & $1.7 \pm 7.0$ & $1.1 \pm 2.5$ & 0.14 \\
\hline Transfusión de GR (n, \%) & $104(23.9 \%)$ & 19 (18.4\%) & 0.23 \\
\hline Estadia intrahospitalaria (dias) & $3[2-7]$ & $3[2-6]$ & 0.07 \\
\hline
\end{tabular}

Mortalidad: La mortalidad fue de $55.2 \%$ ( $\mathrm{n}=214$ pacientes) en el grupo $1, \mathrm{y}$ de $22.3 \%$ ( $\mathrm{n}=19$ pacientes) en el grupo 2, a una mediana de seguimiento de 183[54-409] días y 378 [177-690] días, respectivamente, $\mathrm{p}<0.001$ (Figura 1). Los factores asociados a mortalidad identificados por análisis multivariado fueron VAB como terapia única (HR 2.2, [95\% IC 1.4-3.5], p<0.001), falla renal crónica (HR 2.0, [95\% IC 1.3-3], p <0.001), presión arteria pulmonar (HR 1.02, [95\% IC 1.01-1.03], p=0.002), y presión Aórtica sistólica (HR 0.98, [95\% IC 0.97-0.99], p=0.03).

Éxito de VAB: Las mediciones hemodinámicas estuvieron disponibles en 526 de 538 procedimientos. La tasa global de éxito de BAV fue de $84.3 \%$ ( $n=443)$, similar entre pacientes sometidos a VAB como terapia única $(85.3 \%, \mathrm{n}=361)$ o como puente $(80.4 \%, \mathrm{n}=82), \mathrm{p}=0.21$. Un modelo de regresión logístico incluyendo el período de tratamiento (antes y después del año 2010), tamaño de balón, área valvular aórtica, fracción de eyección y repetición de procedimiento identificó a la repetición de la VAB como el principal factor asociado a falla de VAB (HR 4.34 [95\% IC 2.2-8.3], p <0.001). El incremento medio del área valvular aórtico fue significativamente mayor en 
el primer procedimiento que en VAB sucesivas $(0.4 \pm 0.25$ vs. $0.28 \pm 0.22, \mathrm{p}<0.001)$.

Tasa de complicaciones: Las complicaciones ocurridas peri-procedimiento antes y después del año 2010 son presentadas en la Tabla 4. El compuesto de eventos adversos serios, muerte-ACV ocurrió en 13 procedimientos (4.4\%) antes del 2010 y en 9 intervenciones (3.7\%) después del $2010, p=0.86$. Insuficiencia Aórtica severa después de la VAB fue notada en 6 casos (tasa global de 1.1\%), todas ellas ocurrieron en pacientes con regurgitación basal al menos moderada, en la cual VAB fue usada como medida de salvataje. Complicaciones vasculares ocurrieron menos frecuentemente durante el último período (después del 2010) comparado contra período anterior, $\mathrm{n}=24(8.1 \%)$ vs. $\mathrm{n}=10(4.1 \%), \mathrm{p}=0.05$.

Figura 1: Curva de sobrevida en pacientes sometidos a valvuloplastía Aórtica como terapia única o puente para reemplazo valvular aórtico.

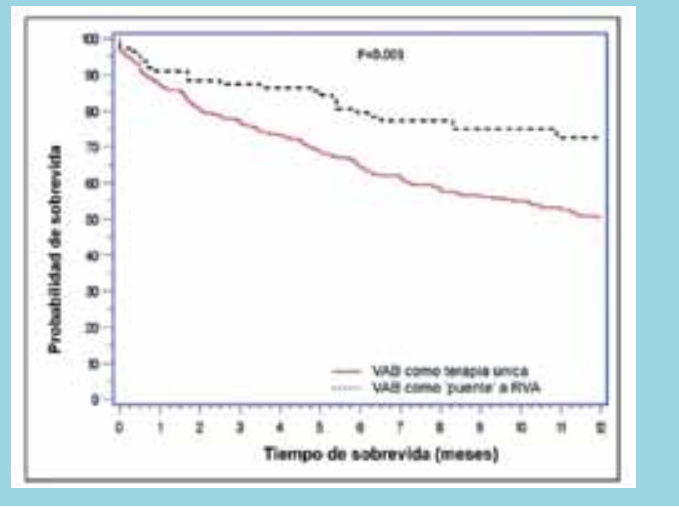

Curva de sobrevida de Kaplan-Meier en pacientes sometidos a valvuloplastía Aórtica con balón como terapia única rojo) y como 'puente' a reemplazo valvular aórtico percutáneo o quirúrgico (negro).

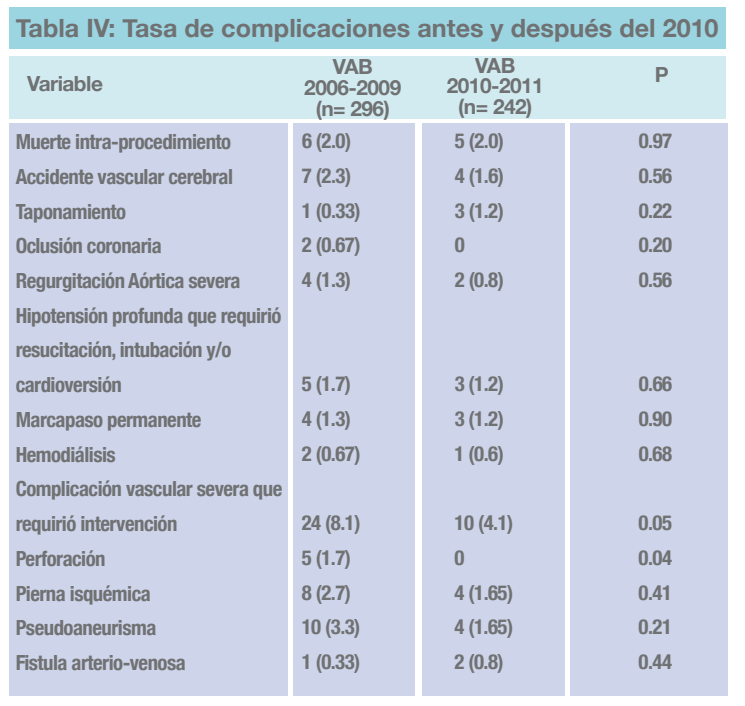

VAB, valvuloplastía Aórtica con balón

\section{Discusión:}

El presente estudio sugiere que en pacientes con EA severa de alto riesgo y contraindicación temporal para RVA quirúrgico/percutáneo la valvulplastía aórtica con balón (VAB) puede ser usada en forma relativamente segura como puente para una intervención definitiva con buenos resultados a mediano plazo. Asímismo este estudio sugiere que en pacientes no candidatos a RVA, la VAB puede ser usada en forma segura pero está asociada a pronóstico pobre.

El uso de VAB como terapia única, la falla renal crónica y el grado de hipertensión pulmonar fueron los principales factores asociados a mortalidad en esta población. Estos factores han sido previamente vinculados a pronóstico adverso en pacientes con EA severa., ${ }^{9,11,12,13} \mathrm{La}$ falta de una terapia definitiva que alivie la obstrucción mecánica impuesta por la EA severa está asociado a pobres resultados. ${ }^{4,5}$ En tanto la falla renal crónica, un potente marcador de comorbilidad y la hipertensión pulmonar, que se condice con el efecto hemodinámico crónico de la EA, resultaron ser los principales cofactores asociados a mortalidad en la población estudiada, lo cual es consistente con reportes previos. ${ }^{11,12,13}$

En relación a los predictores de éxito/falla de VAB, como ya previamente habíamos reportado,,$^{10}$ la repetición del procedimiento de $\mathrm{VAB}$ estuvo asociada a menores tasas de éxito. Se han descrito que la fractura de los depósitos de calcio en los velos valvulares y en menor grado el estiramiento del anillo y la separación de las comisuras fusionadas como los mecanismos que alivian la EA con el uso de la $\mathrm{VAB}^{14}$ Es posible que la re-estenosis sobreimpuesta a una válvula Aórtica severamente calcificada límite el beneficio de sucesivas dilataciones.

La reciente introducción de RVA percutáneo ha reactivado la realización de la VAB como un paso preliminar a RVA percutáneo o quirúrgico. ${ }^{10}$ Sin embargo muchos pacientes referidos para RVA percutáneo no son elegibles para esta terapia, debido a la existencia de comorbilidades o daño cardíaco avanzado ${ }^{15}$. El empleo de la VAB está indicado en la paliación de síntomas en aquellos pacientes considerados inoperables o como puente a un RVA definitivo a fin de estabilizar la condición clínica del paciente, especialmente en la presentación con shock cardiogénico o falla cardíaca descompensada grave. ${ }^{16} \mathrm{El}$ presente estudio sugiere que la VAB puede ser empleada como una herramienta útil de selección para RVA definitivo, como sugerido por estudios previos. ${ }^{17}$ Proponemos el uso de la VAB como una prueba-terapéutica que permita valorar la mejoría clínica de pacientes severamente enfermos, reversibilidad de disfunción VI severa, reducción de insuficiencia mitral o hipertensión pulmonar severa y recuperación 
del debilitamiento y/o fragilidad severa que presentan estos pacientes. Consistentemente con lo antes expuesto, $\mathrm{y}$ en concordancia con previos reportes ${ }^{15,18}$ nuestros resultados apoyan la hipótesis que la preparación con VAB previo a RVA puede ser una efectiva alternativa para reducir la tasa de complicaciones en pacientes con EA severa de alto riesgo, dado que los resultados del RVA percutáneo están fuertemente influenciados por la selección de los candidatos apropiados, evitando el uso del RVA percutáneo en la llamada cohorte 'C' (pacientes en los cuales el beneficio del RVA es mínimo o nulo debido a avanzada comorbilidades).

La presente serie sugiere que la VAB es una técnica relativamente segura, aunque no exenta de riesgos, los cuales parecer ser inherentes a la población tratada. Es importante destacar que la tasa de regurgitación aórtica severa fue baja y ocurrió en pacientes con insuficiencia aórtica basalmente al menos moderada. En nuestra práctica actual no recomendamos el uso de VAB en presencia de insuficiencia aórtica más que moderada o severa. De manera interesante, las mejorías en la técnica y dispositivos no se tradujeron en mejores tasas de éxito de la $\mathrm{VAB}$, pero sí se asociaron a reducción en la tasa de complicaciones vasculares. Dichas mejorías pueden ser atribuibles a el uso de catéteres mas pequeños y a la mejoría en técnicas de cierre percutáneo.

El presente estudio tiene varias limitaciones. Se trata de una serie retrospectiva, no controlada de un sólo centro, por lo cual tiene todas las limitaciones propias de este tipo de estudio. Pese al ajuste por análisis multivariado, factores confundentes o prejuicio de selección pueden haber influenciado los resultados. Los resultados reportados son aplicables a población con EA degenerativa severa de alto riesgo y no deben ser generalizados a poblaciones diferentes.

\section{Conclusión:}

En pacientes de alto riesgo con EA severa y contraindicación temporal para RVA quirúrgico/percutáneo, la VAB puede ser usada como puente para una intervención definitiva con buen resultado a mediano plazo.

\section{Referencias:}

1. LUND O. Preoperative risk evaluation and stratification of longterm survival after valve replacement for aortic stenosis. Reasons for earlier operative intervention. Circulation. 1990; 82:124-39.

2. SMITH CR, LEON MB, MACK MJ, MILLER DC, MOSES JW, SVENSSON LG, et al. Transcatheter versus surgical aorticvalve replacement in high-risk patients. N Engl J Med. 2011; 364:2187-98.

3. LEON MB, SMITH CR, MACK M, MILLER DC, MOSES JW, SVENSSON LG, et al. Transcatheter aortic-valve implantation for aortic stenosis in patients who cannot undergo surgery. $\mathrm{N}$ Engl J Med. 2010; 363:1597-607.

4. BEN-DOR I, PICHARD AD, GONZALEZ MA, WEISSMAN G, LI Y, GOLDSTEIN SA, et al. Correlates and causes of death in patients with severe symptomatic aortic stenosis who are not eligible to participate in a clinical trial of transcatheter aortic valve implantation. Circulation. 2010; 122:S37-42.

5. BAVARIA JE, SZETO WY, ROCHE LA, WALSH EK, BUCKLEY-BLASKOVICH V, SOLOMETO LP, et al. The Progression of a Transcatheter Aortic Valve Program: A Decision Analysis of More Than 680 Patient Referrals. Ann Thorac Surg. 2011
Oct 4. [Epub ahead of print].

6. CRIBIER A, SAVIN T, SAOUDI N, ROCHA P, BERLAND J, LETAC B. Percutaneous transluminal valvuloplasty of acquired aortic stenosis in elderly patients: an alternative to valve replacement? Lancet 1986;1:63-7.

7. Percutaneous balloon aortic valvuloplasty. Acute and 30-day follow-up results in 674 patients from the NHLBI Balloon Valvuloplasty Registry. Circulation 1991;84:2383-97.

8. SAFIAN RD, BERMAN AD, DIVER DJ, MCKAY LL, COME PC, RILEY MF, et al. Cunningham MJ, Wyman RM, Weinstein JS, et al. Balloon aortic valvuloplasty in 170 consecutive patients. N Engl J Med 1988;319:125-30.

9. OTTO CM, MICKEL MC, KENNEDY JW,ALDERMAN EL, BASHORE TM, BLOCK PC, et al. Three-year outcome after balloon aortic valvuloplasty. Insights into prognosis of valvular aortic stenosis. Circulation 1994;89:642-50.

10. BEN-DOR I, PICHARD AD, SATLER LF, GOLDSTEIN SA, SYED AI, GAGLIA MA JR, et al. Complications and outcome of balloon aortic valvuloplasty in high-risk or inoperable patients. JACC Cardiovasc Interv 2010; 3:1150-1156.

11. GROSSI EA, SCHWARTZ CF, YU PJ, JORDE UP, CROOKE GA, GRAU JB, et al. High-risk aortic valve replacement: are the outcomes as bad as predicted? Ann Thorac Surg 2008;85:102-6. 
12. DING WH, LAM YY, DUNCAN A, LI W, LIM E, KAYA MG, et al. Predictors of survival after aortic valve replacement in patients with low-flow and high-gradient aortic stenosis. Eur J Heart Fail 2009;11:897-902.

13. SHERMAN W, HERSHMAN R, LAZZAM C, COHEN M, AMBROSE J, GORLIN R. Balloon valvuloplasty in adult aortic stenosis: determinants of clinical outcome. Ann Intern Med 1989;110:421-5

14. ISNER JM, SAMUELS DA, SLOVENKAI GA, HALABURKA KR, HOUGEN TJ, DESNOYERS MR, et al. Mechanism of aortic balloon valvuloplasty: fracture of valvular calcific deposits. Ann Intern Med 1988;108:377- 80.

15. BEN-DOR I, PICHARD AD, SATLER LF, OKUBAGZI P, TORGUSON R, XUEZ, et al. Clinical profile, treatment assignment and clinical outcome of patients with severe aortic stenosis not eligible to participate in a clinical trial of percutaneous aortic valve replacement. Am J Cardiol. 2010 Mar 15;105:857-61.

16. BONOW RO, CARABELLO BA, CHATTERJEE K, DE LEON AC JR, FAXON DP, FREED MD, et al; American Co- llege of Cardiology/American Heart Association Task Force on Practice Guidelines. 2008 focused update incorporated into the ACC/AHA 2006 guidelines for the management of patients with valvular heart disease: a report of the American College of Cardiology/American Heart Association Task Force on Practice Guidelines (Writing Committee to revise the 1998 guidelines for the management of patients with valvular heart disease). Endorsed by the Society of Cardiovascular Anesthesiologists, Society for Cardiovascular Angiography and Interventions, and Society of Thoracic Surgeons. J Am Coll Cardiol. 2008 Sep 23;52(13):e1-142.

17. SAIAF, MARROZZINI C, MORETTI C, CIUCAC, TAGLIERI N, BORDONI B, et al. The role of percutaneous balloon aortic valvuloplasty as a bridge for transcatheter aortic valve implantation.EuroIntervention. 2011 Oct 30;7:723-9.

18. USSIA GP, CAPODANNO D, BARBANTI M, SCARABELLI M, IMME S, CAMMALLERI V, et al. Balloon aortic valvuloplasty for severe aortic stenosis as a bridge to high- risk transcatheter aortic valve implantation. J Invasive Cardiol 2010;22:161-6. 